PROFESSOR GRAEME D RUXTON (Orcid ID : 0000-0001-8943-6609)

Article type : Research Papers

\title{
The evolutionary stability of attenuators that mask information about animals that social partners can exploit
}

Corresponding author mail-id :gr41@st-andrews.ac.uk

Sean Hackett ${ }^{1}$ \& Graeme D Ruxton ${ }^{2}$

1. Department of Zoology, University of Oxford, Oxford OX1 3PS, UK

2. School of Biology, University of St Andrews, St Andrews KY16 9TH, UK

\section{Abstract}

Signals and cues are fundamental to social interactions. A well-established concept in the study of animal communication is an amplifier, defined as a trait that does not add extra information to that already present in the original cue or signal, but rather enhances the fidelity with which variation in the original cue or signal is correctly perceived. Attenuators as the logical compliment of amplifiers: attenuators act to reduce the fidelity with which variation in a signal or cue can be reliably evaluated by the perceivers. Where amplifiers reduce the effect of noise on the perception of variation,

This article has been accepted for publication and undergone full peer review but has not been through the copyediting, typesetting, pagination and proofreading process, which may lead to differences between this version and the Version of Record. Please cite this article as doi: $10.1111 /$ jeb. 13253

This article is protected by copyright. All rights reserved. 
attenuators add noise. Attenuators have been subject to much less consideration than amplifiers, however they will be the focus of our theoretical study. We utilise an extension of a well-established model incorporated signal or cue inaccuracy and costly investments by emitter and perceiver in sending and attending to the signal or cue. We present broad conditions involving some conflict of interest between emitter and perceiver where it may be advantageous for emitters to invest in costly attenuators to mask cues from potential perceivers, and a subset of these conditions where the perceiver may be willing to invest in costly anti-attentuators to mitigate the loss of information to them. We demonstrate that attenuators can be evolutionary stable even if they are costly, even if they are sometimes disadvantageous, and even if a perceiver can mount counter-measures to them. As such, we feel that attenuators of cues may be deserving of much more research attention.

Keywords: signalling, communication, cues, amplifiers, costly signals

\section{Introduction}

Interactions between organisms often involve the behaviour of individuals being influenced through stimulation of their senses by traits of other individuals. That is, the behaviour of individuals is responsive to sensory stimulations triggered by either unselected cues, produced by other individuals, or signals, whose form has been influenced in part by the effect they have on the sensory systems of others. Such communication has been extensively studied (see Maynard Smith \& Harper 2003; Searcy \& Nowicki 2005; Bradbury \& 
Vehrencamp 2011 for reviews), and is fundamental to processes such as mate choice, parental care, and diet choice.

Let us call the individual that bears the traits the emitter, and the individual whose sensory systems are stimulated by those traits in a way that influences its subsequent behaviour, the perceiver. Of course, an individual can switch between these roles, or even play both roles simultaneously, but there is no loss of generality from our focus on the simple case of fixed roles. If the two individuals are interacting, then the nature of the traits expressed by the emitter can have a direct bearing on its fitness, for example influencing whether or not the perceiver attempts to mate with it, feed it, or attack it. This concept has been at the heart of our understanding of how signals can evolve for the primary purpose of influencing the behaviour of others (e.g. such as exaggerated features or coloration used in mate choice).

One aspect of such communication is the concept of an amplifier. This concept was introduced by Hasson (1989). Consider an example situation where there is a cue or signal that females use to select which males to mate with. This could be a simple physical characteristic, such as body size, or in the form of a sexual ornament, such as: antlers, tusks, horns, feathers, tail patterns, or other ornamental colouration. There must clearly be variation in the detected trait that allows females to differentiate between males. It is likely that the trait that stimulates their senses is correlated with traits that are linked to quality; that is, the variation in the trait is informative about variation in males in terms of their quality as a mate. For example, in red jungle fowl (Gallus gallus), females have been found to select males based on eye colour and comb size and colour; these traits are associated with mate condition and parasite resistance (Zuk et al. 1990a, b, c, d). Hasson introduced the concept of an amplifier as a trait that did not add extra information to that already present in the original cue or signal, but rather enhanced the fidelity with which variation in the original cue or signal was correctly perceived. For example, it might be that red colouration on the breast of 
a bird is a cue or signal used by females, who prefer males with a greater extent of red on the breast. If all males adopt a courtship dance which involves displaying their breast prominently to females, then this can be considered an amplifier. The dance is performed identically by all males, so the dance itself is not a means of differentiating between males; however, the dance allows females to evaluate the extent of red colouration on the breast more reliably. Such amplifiers are an accepted aspect of the evolution of signal form (e.g. Maynard Smith \& Harper 2003) and their importance to the evolution of signals has recently been subject of careful theoretical investigation (e.g. Hackett et al. 2016, Bogaardt \& Johnstone 2016). However, Hasson et al. (1992) introduced attenuators as the logical complement to amplifiers: attenuators act to reduce the fidelity with which variation in a signal or cue can be reliably evaluated by the perceivers. Where amplifiers reduce the effect of noise on the perception of variation, attenuators add noise. Attenuators have been subject to much less consideration than amplifiers. Essentially, the concept of attenuators where introduced as the logical complement of amplifiers by Hasson (1989), although he did not explore evolutionary aspects of such traits. Since then the only development was that of Bogaardt \& Johnstone (2016) demonstrated that amplifiers (and thus by analogy attentuators) can easily evolve to become informative and become a signal in their own right. Here we will focus on discussing the circumstances that should select for the evolution and maintenance of attentuators, and also of countermeasures against them (which we term "anti-attentuators").

Emitters might be selected to use an attenuator (even if it is costly to produce) if there is conflict of interest (at least sometimes) between the emitter and perceiver. That is, if the accurate evaluation of the cue or signal causes the perceiver to act in a way that imposes a cost on the emitter, then emitters may be selected to reduce the accuracy of such a cue or signal. 
An example of this might be seen in the interaction between great tits (Parus major) and pied flycatchers (Ficedula hypoleuca) in a selection of nest boxes at various sites in Finland, studied by Jukka Forsman and collaborators (see Loukola et al. 2014 and references therein). Resident great tits select nest boxes and begin nesting before the arrival of migratory flycatchers. It has been shown that flycatchers inspect nest boxes and preferentially take up residence in vacant nest boxes near to those occupied by great tits that have produced large clutches of eggs. This may be adaptive if locally large great tit clutches are indicative of locally high food availability. Such inspection involves a cost to flycatchers, not just in time and energy but also an injury or even mortality risk if they are discovered in a nest box by the returning resident great tit. Further, there is a cost to great tits when flycatchers nest nearby, expressed as reduced fledging success, and likely driven by local competition between tits and flycatchers for food to feed chicks. Loukola et al. (2014) showed that great tits covered their eggs with nest material each time they departed from the nest box when subject to experimental treatments designed to indicate presence of flycatchers, but not in a control treatment involving another bird species. The authors conclude that this covering behaviour acts to deprive the flycatchers of information about clutch size. Although this has yet to be demonstrated conclusively, it does seem the most plausible explanation. In terms introduced earlier, we see clutch size as a cue produced by great tits that provides useful information to flycatchers about food quality in the local environment. High food quality in the area will lead to successful foraging by great tits, better quality reproductive individuals, and result in greater reproductive rates, evidenced by clutch size. This information is acted upon by flycatchers in their nest site choices, and the covering behaviour by the great tits is a trait that reduces the fidelity of the information available to the flycatchers.

This article is protected by copyright. All rights reserved. 
In general, we would expect that attenuators will often be costly for the emitter, and grow more costly the more they erode signal or cue accuracy. In the nest box example, there are time and energy costs associated with bringing extra nesting material into the box for the purpose of egg covering (as observed by Loukala et al. 2014), and in the effort taken to cover the eggs prior to departure and uncover them again later; the eggs must be uncovered again in order for contact-incubation, where parents' brood patches must be directly contacting eggs to regulate heat transfer and monitor temperatures (Boulton \& Cassey, 2012; DuRant et al., 2013). The greater the investment in these covering behaviours, the more fully the eggs can be covered, and the more effective the attenuator is in reducing the informational content of the cue, but there will be diminishing returns on ever greater investment. Sometimes the perceiver may be able to evolve what we term anti-attenuators that reduce the effectiveness of attenuators. For example, Loukola et al. (2014) report that flycatchers will enter nest boxes and remove material from the top of the covered eggs. We would expect that there is a cost to anti-attenuators, in general, that increases with the extent to which they reduce noise added by the attenuator. In the nest box example, given enough time investment, a flycatcher can likely fully uncover eggs no matter how deeply they are buried, but the more time it spends in such activity, the greater the time, energy and mortality costs.

Our aim in this paper is to explore the circumstances under which emitters will invest in attenuators and perceivers in anti-attenuators. To explore the issues described above, we utilise an extension of the well-established model of Bradbury \& Vehrencamp (2000). Their model incorporated signal or cue inaccuracy and costly investments by emitter and perceiver in sending and attending to the signal or cue. In previous papers (Hackett et al. 2014, 2016), we introduced a functional linkage between these two issues, and assumed that while both emitter and perceiver can exert some control over signal or cue fidelity, increasing levels of control are increasingly costly. However, in our previous work we assumed that investment 
by either party only served to improve signal or cue fidelity, via amplifiers. Here we are interested in the fundamentally different case where we expect emitters to potentially deploy attenuators that erode the fidelity with which perceivers detect variation in the form of a cue.

\section{Methods}

The core structure of the model remains unchanged from that of Bradbury and Vehrencamp (2000) and Hackett et al. (2014, 2016). A perceiver is subject to one of two mutually-exclusive conditions $\left(C_{1}\right.$ or $\left.C_{2}\right)$ and may choose to adopt one of two possible actions $\left(A_{1}\right.$ or $\left.A_{2}\right)$. We assume that condition $C_{1}$ occurs with probability $P_{1}$ and condition $C_{2}$ occurs with probability $P_{2}=1-P_{1}$. The payoff to the perceiver for selecting action $i$ when condition $j$ applies is $W_{i j}$. We further assume that $A_{1}$ is the optimal action when $C_{1}$ applies $\left(W_{11}>W_{21}\right)$ and define $\Delta W_{1}$ as $W_{11}-W_{21}$ as the benefit of taking the optimal action when condition 1 pertains; while $A_{2}$ is the optimal action in $C_{2}\left(W_{22}>W_{12}\right)$ with $\Delta W_{2}$ being defined similarly. We anticipate that an optimal perceiver will always try to select the course of action that maximises its fitness. Yet, the perceiver does not know which condition applies and would thus benefit from information relating to the prevailing condition. The emitter has information about the prevailing condition, and that information is available to the perceiver in the form of a cue produced by the emitter (in the absence of investment in an attenuator by the emitter). However, this cue might not provide unambiguous information on prevailing conditions. These assumptions mirror those of Hackett et al. $(2014,2016)$. The key difference in the model explored here, is that we are interested in situations where there is conflict of interest between perceiver and emitter in at least one of the conditions, such that the cue is potentially disadvantageous to the emitter. Further,, the emitter might have a means to invest in an attenuator that reduces the informational value of the cue; but the perceiver can counter this with investment in an anti-attenuator that mitigates the effects of the attenuator.

This article is protected by copyright. All rights reserved. 
As in Hackett et al. $(2014,2016)$., the payoff to the emitter when the perceiver selects action $i$ in condition $j$ is $T_{i j}$. If $T_{11}>T_{21}$ and $T_{22}>T_{12}$ then the interests of the sender and perceiver are always congruent, otherwise there is at least a partial conflict of interest. $\Delta T_{i}$ is the benefit to the emitter of the perceiver taking the emitter's preferred action when condition $i$ pertains. Just as the perceiver seeks to maximise its fitness by minimising losses - of, for example, time and energy - due to incorrect decisions, the emitter aims to maximise its fitness by influencing the decision-making processes of the perceiver such that the perceiver selects the emitter's preferred action for the current condition.

One barrier to effective influencing by the emitter may be an instance where the perceiver changes its behaviour in a way that costs the emitter as a result of information received in the cue. In order to reduce such costs, the emitter could invest in attenuation of the cue. The emitter pays costs in order to attenuate the cue, with increasing levels of attenuating being increasingly costly but also increasingly effective in reducing the influence of the cue on the perceiver. Attenuators thus need to outweigh these costs by changing the responses of the perceiver with sufficient frequency in ways that benefit the emitter. We assume that the emitter always produces a cue, but may or may not additionally invest in an attenuator. The description of investment in these attenuators by the emitter and in antiattenuation traits by the perceiver are the only ways in which the model here deviates from that of Hackett et al. $(2014,2016)$.

The accuracy of the cue (as perceived) is denoted by $Q$ representing the conditional probability that the perceiver will interpret the cue as describing the correct condition and therefore perform the most beneficial action for itself under this condition. We retain the simplifying assumption of Bradbury and Vehrencamp (2000) that the accuracy values are identical in each condition. Since the entity of interest to the perceiver has two levels $\left(C_{l}\right.$ and 
$\left.C_{2}\right)$, we can expect that the emitter can produce one of two possible cues $\left(S_{1}\right.$ or $\left.S_{2}\right)$, with some (generally less than perfect) association between the specific levels of $C$ and $S$. Since these are arbitrary, we can assume without any loss of generality that (in the mind of the perceiver) $S_{1}$ is associated with $C_{1}$ and $S_{2}$ with $C_{2}$. In the presence of cues, the perceiver detects the correct cue $\left(S_{1}\right.$ in $C_{1}$ and $S_{2}$ in $C_{2}$ ) with probability $Q$, and the incorrect cue with probability 1-Q. Higher values of $Q$ represent more accurate information. For a cue to be useful to the perceiver, $Q$ must assume a value greater than 0.5 (which represents chance for a binary choice).

In the absence of attending to the cue, the perceiver is expected to assume that one default condition $\left(C_{1}\right.$ or $\left.C_{2}\right)$ always pertains (we call this the insular strategy; and, for the parameter values used throughout this study, this strategy is to assume that $C_{1}$ always applies). Attending to cues has the potential to allow the perceiver some ability to correctly identify situations where the non-default condition $\left(C_{2}\right)$ pertains. However, attending to the cue also introduces the risk of the perceiver mistakenly acting as if the non-default condition $\left(C_{2}\right)$ pertains in cases where the insular strategy would have caused it to take the best behavioural option. Crucially, the benefits of attending to cues must outweigh the cost of errors. Thus, there are two major costs for the perceiver of attending to the cue under this framework. First is the efficiency cost of any investment in anti-attenuators. Second is the cost incurred by the decrease in the rate of correct decisions in the default condition as a consequence of imperfect coding (either the wrong cue has been transmitted and/or the correct cue has been misunderstood). We call the perceiver strategy that involves acting as if the condition suggested by the state of the cue pertains the conditional strategy.

Note that it does not always pay for the perceiver to alter its behaviour conditionally depending on the perceived cue. For instance, if the cue is fairly unreliable, then the perceiver should consistently do whatever has the highest average payoff (i.e. what we call the 'insular 
strategy'). Clearly, if the intrinsic informational value of the cue is insufficient to cause the perceiver to give up on the insular strategy of always assuming condition $C_{l}$ in favour of a strategy that is conditional on the cue, then there is no reason for the emitter to invest in reducing the informational content of the cue. Hence, we are particularly interested in combinations of parameters where (in the absence of investment by the emitter) is pays the perceiver to modify its behaviour in the light of the cue. If investment in anti-attenuators by the perceiver is given by $K_{p}$, then a conditional strategy is superior to the insular strategy for the perceiver if and only if both of the following inequalities hold:

$$
\begin{aligned}
& \left(1-P_{1}\right) Q \Delta W_{2}-P_{l}(1-Q) \Delta W_{l}-K_{p}>0 \\
& P_{l} Q \Delta W_{l}-\left(1-P_{l}\right)(1-Q) \Delta W_{2}-K_{p}>0
\end{aligned}
$$

The payoff to the insular strategy is $\left(P_{1} W_{11}+P_{2} W_{12}\right)$ if its default action is $A_{l}$. The payoff to the conditional strategy is $\left(P_{1} Q W_{11}+P_{2} Q W_{22}+P_{1}(1-Q) W_{21}+P_{2}(1-Q) W_{12}-K_{p}\right)$. Subtracting the insular payoff from the conditional one gives the condition for conditional one to be favoured. The second condition is obtained in an analogous way, but using a resident population with a default action $A_{2}$. Generally, one of the insular strategies will be superior to the other. Without loss of generality, in this paper, we always select parameter values such that always taking action $A_{1}$ is superior to always playing $A_{2}$, and thus satisfying the first condition above will automatically mean the second condition is satisfied. Intuitively, the first condition will fail, and anti-attenuating traits will be selected against, whenever the cost of the traits, $K_{p}$, exceeds the benefits they confer. We separate the fidelity of the cue in reliably informing about the underlying state into two components: the fidelity of the cue as emitted $(q)$; and the fidelity of the signal as perceived $(Q)$. The first of these is a maximum value for the second, assuming perfect fidelity of transmission through the environment and perfect 
detection by the perceiver. The fidelity of a cue as it is emitted, is necessarily a function of investment $\left(K_{e}\right)$ by the emitter in attenuators, Specifically:

$q=0.5+(Z-0.5) \exp \left(-\alpha K_{e}\right)$

where $\mathrm{Z}$ describes the intrinsic informational value of the cue (in the absence of investment in attenuators) and may take any value from the range $0.5<Z \leq 1$. The positive constant $\alpha$ now represents the relative ease with which informational content of the cue is suppressed. The greater the value of $\alpha$, the greater the decrease in the emitted fidelity of a cue that can be bought for a given level of investment. When there is no investment in attenuations $\left(K_{e}=0\right)$ then $q=Z$. With increasing investment in an attenuator (increasing value of the positive parameter $K_{e}$ ), the value of q declines, asymptoting at the completely uninformative state $q=$ 0.5 .

However, if $K_{p}$ is the perceiver's investment in an anti-attenuator then

$Q=q+(Z-q)\left(1-\exp \left(-\beta K_{p}\right)\right)$

where $\beta$ is a positive constant which encapsulates the relative ease with which investment in an anti-attenuator improves fidelity of the cue. In the absence of investment in an antiattenuator by the perceiver (i.e. when $K p=0$ ), then $\mathrm{Q}=\mathrm{q}$ and the fidelity of the cue as perceived is exactly as it was emitted by the other party. However, as $K_{p}$ increases so $Q$ increases, although this effect saturates and $Q$ is bounded above at the value $Z$. Thus, no matter how heavily the emitter invests in attenuators (i.e. no matter how near $q$ is to 0.5 ), the perceiver can recover up to the full informational content of the cue $(Z)$ providing it is prepared to invest sufficiently in anti-attenuators. We adopted exponential forms for these functions to represent a situation common in biology and beyond where there are diminishing returns on increasing levels of investment. As we revisit in the Discussion, we do not except 
our qualitative conclusions to be changed by modification of the shape of these functions (for example) linear or sigmoidal forms - and we leave such elaborations to more system-specific developments of the methodology presented here.

It is worth noting that the zero equilibrium of the model, in which neither party invests in altering cue fidelity, is stable. As this equilibrium is dynamically uninteresting, we instead opt to displace the model from this equilibrium by initiating simulations using the above function for detected signal fidelity from a point in which one party (the emitter) already makes a small investment (it does not matter which one is selected).

We simulated the dynamics of the model in $R$ (version 3.3.3) for a range of conditional probabilities and parameter values. Simulations were run for 500 generations, and at each generation one of either $K_{e}$ or $K_{p}$ was randomly selected for mutation. Mutations took the form of the addition or subtraction of a randomly-generated number selected from a normal distribution $(\mu=0, s d=0.025)$. Where this resulted in either $K_{e}$ or $K_{\mathrm{p}}$ assuming a negative value, the change was discarded and another mutation was generated. Since it is only $Q$ that takes a different value for mutants and residents, their relative fitness is dependent only on terms proportional to $Q$. Mutations that improved the fitness of the selected party were retained and provided the basis for subsequent mutations in future generations. Fitness values were calculated as follows:

$W_{p}=Q\left(P_{1} \Delta W_{1}+\left(1-P_{1}\right) \Delta W_{2}\right)-K_{p}$

$W_{s}=Q\left(P_{1} \Delta T_{1}+\left(1-P_{1}\right) \Delta T_{2}\right)-K e$,

Conversely, mutations which decreased the fitness of the selected party were rejected (this is a similar approach to used in adaptive dynamics: Diekmann2002). We continued this process until an equilibrium was identified or 4000 generations had elapsed. Specifically, from the 
$550^{\text {th }}$ generation onwards, the mean investment values for each party over the preceding 25 generations were computed and compared to those of the 25 generations which in turn preceded them. If the mean investment of both parties was found to have remained unchanged over the focal 50 generation period, then the model was deemed to be at equilibrium. If an equilibrium was not identified within 4000 generations, often attributable to rapid antagonistic cycles of investment between the parties (an example of which is illustrated in Figure S1), then equilibrium values were approximated by taking their mean between the $500^{\text {th }}$ generation and the $4000^{\text {th }}$ generation. We exclude the initial 500 generations to account for instances where the pertinent values for each party (investment, fitness and perceived/emitted fidelity) deviated substantially from their initial conditions before either attaining a fixed equilibrium or oscillating around an equilibrium. Further details and derivation can be found in Hackett et al. (2014, 2016).

We explore two different situations: (i) the two parties disagree on the perceiver behaviour they would prefer only when only one condition $\left(C_{1}\right.$ or $\left.C_{2}\right)$ applies and (ii) they always disagree.

\section{Linking the model to the avian nesting example}

In the avian nesting example, the two potential conditions are that the tit has nested in either a good-quality or poor-quality territory. The cue relating to territory quality will be clutch size: large for a good-quality territory or small for a poor-quality territory. The options open to the flycatcher are either to share the territory with the tit or to move on and avoid sharing the territory. The flycatcher benefits from finding and sharing a goodquality territory, but the optimal behaviour from its perspective if it correctly perceives the territory to be poor quality, is to depart and seek a good-quality territory elsewhere. 
In some circumstances, the behaviour that benefits the flycatcher might also benefit the tit. That is, when the territory is poor quality then the tit might benefit from communicating this accurately to the flycatcher so the flycatcher departs, and the tit might also benefit from the flycatcher sharing its territory if the territory is good quality. This latter circumstance might occur if there is no real competition cost in a good-quality territory and there is some other benefit to the tit (for example, through dilution of risk of predation or collective defence against predation) from a flycatcher nesting nearby. In general, where the interests of the two parties always align then we would not expect attenuators or anti-attenuators to evolve (even if such adaptations are cost free).

Next, we could imagine a situation where there is agreement in one situation but disagreement in the other. For example, the tit might always benefit if the flycatcher departs, but the flycatcher might do best by sharing when the territory is high quality but departing when the territory is poor quality. In this situation, there is agreement when the territory is low quality but disagreement when the territory is high quality. In such a situation, it might be possible to imagine a costly attenuator evolving, but only if the attenuator cannot be used selectively or when the flycatcher cannot detect the use of the attenuator. That is, when the egg-burying behaviour is not used only by tits in high-quality patches, or when the flycatcher cannot easily differentiate between a nest with buried eggs and a nest in which eggs have yet to be laid. Imagine a circumstance where tits bury eggs all the time. A mutant that only buried its eggs when the territory was good quality would benefit, because it would save costs of egg-burying and it would allow flycatchers to accurately see when it has laid a small clutch as an indicator of a low-quality patch. Thus, such mutants would spread through the population. But when 
such mutants become widespread, then the benefit of egg-burying to tits is lost, because egg burial becomes a reliable cue of a high-quality territory to the flycatchers. In this situation, tits should be selected to give up paying the costs of burial. More generally, we would only expect use of attenuators in situations where there is partial conflict between the signaller and perceiver, in cases where the use of the attenuator does not itself become a reliable indicator of the condition it is selected to mask. Such reliable indication might be avoided if either (i) the perceiver cannot detect that the attenuator has been used and/or (ii) the attenuator cannot be used selectively according to the state of the trait that the perceiver is interested in.

One can also imagine that the tit and flycatcher could agree on the course of action for high-quality patches but not for low-quality patches. This could occur where the tit always benefits from the flycatcher nesting nearby and sharing the territory, while the flycatcher does best by remaining in a good-quality territory but departing from a poorquality one.

We could imagine that the insular behaviour of the flycatcher which ignores information on territory quality from tit-clutch size could be to always depart from territories in which tits are nesting or always try to nest there. Either of these could be plausible - if competition is important in all but the highest-quality territories then (in the absence of further information) avoiding settling near a nesting tit might be a rational strategy; whereas if collective defence from predators is important, or tit nesting is a sign of a reasonably suitable local environment, then always nesting in close proximity could also be a plausible strategy in the absence of information on specific territory quality gained through observing tit-clutch size. 
In terms of the costs and benefits of attenuators and anti-attenuators, the simplest model might be to consider that the cost paid by the tits reflects the fraction of occasions when it leaves the nest having buried the eggs, and burial deprives any flycatcher that looks in the box of any information on clutch size. The higher the cost paid by the tit, the more frequently they bury, and the more effectively they mask this cue. Flycatchers then make repeated visits to the box until they visit when the adults are not there but the eggs have not been buried and learn the true clutch size; the higher the investment the tit makes in burial, the higher the cost the flycatcher will have to pay in repeated visits to the nest to recover the information on clutch size. This cost of antiattenuators - in time and energy (and perhaps exposure to attack) - in repeated visits to the box, is more straightforward to apply than costs based on active uncovering of the eggs. Such uncovering behaviour will yield information in a relatively-complex manner, depending of the cognitive functioning of the flycatcher and the ease with which behaviour uncovers eggs.

In general, it is also possible to imagine circumstances where there is conflict in both states. In the tit-flycatcher system, this might apply to a situation where if the territory is high quality then the tit would like to monopolise it, but it would prefer to share a poor-quality territory with the flycatcher; whereas, the flycatcher would benefit from nesting with a tit only in a high-quality territory. This seems relatively unlikely for this system. The flycatcher cost-benefits in this scenario are highly plausible - it is the situation for the tit that is more problematic. It is just possible to envisage a situation where in a low-quality territory, the tit benefits strongly from collective defence against predators, because the tit must often be away from the nest itself searching for food, whereas in a high-quality territory this benefit is less strong and the main concern for 
the tit is avoidance of competition (or perhaps avoidance of the flycatcher's activity attracting predators into the vicinity). This seems rather far-fetched for this system, but there is still benefit in studying this complete-conflict case in generality.

\section{Results}

In what follows we present equilibrium investment in attenuation $K_{e}$ and antiattenuation $K_{p}$ and the perceived fidelity of the cue $Q$ for two conflict scenarios: conflict in a single condition only and conflict in both conditions (total conflict). Results are presented for three values of the intrinsic informational value of the cue $Z(0.6,0.75 \&$ 0.9). Given that a similar functional form was observed in the equilibrium values of perceiver and emitter investment for all three values of $Z$ for each parameterisation we directly present only results for simulations with $Z=0.75$ here while results for simulations with $Z=0.6$ and $Z=0.9$ are available in the Supplementary Materials. For each value of $Z$, simulations were repeated for three values of $\beta(1,3 \& 5)$ and eleven values of $\alpha$ (from 0 to 5 in increments of 0.5 ), where $\alpha$ and $\beta$ respectively determine the ease of cue attenuation and detection. Unless otherwise stated, the perceiver payoffs are $\Delta W_{1}=\Delta W_{2}=8$ and the default condition occurs with probability $p_{1}=0.8$.

\section{Conflict in one condition}

Figure 1 depicts equilibrium investment $K_{i}$ by each party (Fig.1a) and the perceived fidelity of the cue $Q$ (Fig.1b) for a conflict in the default condition where the emitter payoffs are $\Delta T_{1}=-8, \Delta T_{2}=8$ and $Z=0.75$. Equilibrium investment and perceived cue fidelity for $Z=0.6$ and $Z=0.9$ are provided in Figures S2 and S3 respectively. Equilibrium investment by each party, and the range of $\alpha$ values over which evolution 
occurs, increases as the intrinsic informational value of the cue $Z$ increases. The greater the correspondence between the focal cue and the conflict condition, the greater the selection on the emitter to suppress the information, which in turn selects for antiattenuation by the perceiver. For a given combination of $Z$ and $\beta$ there is a range of $\alpha$ values over which emitters will evolve attenuation but perceivers are not selected to retaliate. The width of this range contracts as $Z$ and $\beta$ are increased and perceivers evolve anti-attenuating traits more readily. Thus, for a given parameterisation, there is some threshold level of attenuation which is beneficial to the emitter but which can be tolerated by the perceiver. That is, for levels of attenuation below this threshold, antiattenuation is not economical for perceivers. When $\alpha$ is great enough that the emitter can either attain or surpass this attenuation threshold, anti-attenuation becomes profitable to the perceiver. Intuitively, it follows that the greater the payoffs available to the perceiver in the conflict condition, the lower the values of $\alpha$ and $\beta$ required to promote perceiver evolution and the less tolerant of any level of attenuation the perceiver becomes (see Fig.5).

Over the range of conditions for which both parties evolve to non-zero investment, the equilibrium value of $Q$ remains fixed at some value which represents a compromise between the incentives of each party and is sensitive to both $Z$ and $\beta$. The sensitivity to $Z$ is intuitive, the greater the value of $Z$, the greater the effort required by the emitter to attenuate the fidelity of the cue to low values. That is, the emitter's preferred value of $Q$ is a reflection of its payoffs, which determine how much is at stake and how much the emitter can afford to spend before attenuation becomes unprofitable. For a given set of payoffs, larger values of $Z$ ensure the emitter reaches this tipping point at a greater value of $Q$ (Fig.S3). Conversely, the lower the value of $Z$, the closer the emitter can 
theoretically drive $Q$ towards the minimum value of 0.5 before further attenuation ceases to be beneficial (assuming no retaliation by the perceiver, Fig.S2). The value of $\beta$ determines the influence of the perceiver. As $\beta$ increases, the cost-efficiency of antiattenuation increases and the perceiver will evolve anti-attenuating traits for lower values of $\alpha$. That is, when effective anti-attenuation requires only a small investment the perceiver is less tolerant of attenuation. Thus as $\beta$ increases, emitter investment decreases and the equilibrium value of $Q$ tends towards $Z$.

When the emitter evolves attenuation in isolation, its investment increases as $\alpha$ increases and the equilibrium value of $Q$ tends towards the emitter's preferred value. Once $\alpha$ becomes sufficiently large that the perceiver is selected to counter the attenuation, emitter investment decreases with further increases to $\alpha$. In contrast, for given values of $Z$ and $\beta$, perceiver investment increases with increasing $\alpha$. When both parties can co-evolve, increasing $\alpha$ does not change the equilibrium value of $Q$; the equilibrium value of $Q$ is now specified by $Z$ and $\beta$ as detailed above. Thus, if antiattenuation serves to prevent further attenuation of $Q$, the changes in the investment of each party as $\alpha$ increases and the environment becomes more conducive to attenuation can be understood in terms of cost efficiency. Greater values of $\alpha$ allow the emitter to 'purchase' greater attenuation for less. Thus, if $Q$ is to be further attenuated and consequently significantly impact perceiver performance, the perceiver is committed to increasing investment in anti-attenuation to counter the improved spending power of the emitter. It follows that, if $\alpha$ enables the emitter to achieve more for less and the antagonism of the perceiver renders further decreases to $Q$ difficult, then the emitter can improve its fitness by investing less to achieve the same outcome. Put simply, anti- 
attenuation imposes a ceiling on emitter investment; the more easily the emitter can attenuate the cue, the harder the perceiver must work to maintain this ceiling.

The frequency of the conflict condition is a key factor in facilitating the evolution of attenuation. If the conflict occurs in the less frequent non-default condition, the potential costs to the emitter must be extremely high and the focal cue sufficiently informative for attenuation to evolve. This is illustrated in Figure 2, which shows equilibrium investment by each party and the perceived fidelity of the cue when the emitter payoffs are $\Delta T_{1}=8, \Delta T_{2}=-40$ and $Z=0.75$. Values for $Z=0.6$ and $Z=0.9$ are shown in Figures S4 and S5 respectively. There is no evolution when $Z=0.6$ (Fig.S4 and where evolution does occur, it is of a lesser magnitude and for a narrower range of $\alpha$ values than observed for a conflict in the default condition (c.f. Fig.1 and Fig.2). For $Z=0.6$ the high cost to the emitter in the conflict condition is mitigated by both the condition's rarity and the relative unreliability of the cue. That is, even if the conflict condition occurs and the cue can be perceived, the perceiver is still likely to select the emitter's preferred action given that the correlation between the cue and the prevailing condition is weak, being only slightly better than random. The infrequency of the conflict also buffers the emitter for larger values of $Z$ where attenuation is favoured, selecting only for low levels of attenuation. Otherwise, when evolution does occur, the same qualitative outcomes are observed as for conflict in the default condition. Notably, irrespective of the change to the emitter payoffs, the equilibrium value of $Q$ when reciprocal evolution between both parties occurs is not significantly reduced (c.f. Fig.1b and Fig.2b). This reinforces the point that it is the perceiver payoffs and their interaction with $Z$ and $\beta$ which dictate the point at which reciprocal investment occurs; 
the emitter payoffs instead act in conjunction with the value of $\alpha$ and $Z$ to determine whether attenuation is beneficial to the emitter in the first instance.

\section{Conflict in both conditions}

A total conflict scenario, in which the parties disagree with respect to the best perceiver action in both conditions, increases equilibrium investment by both parties and extends the range of $\alpha$ values over which evolution occurs. However, the observed trends are otherwise similar to those reported for conflict in the default condition, and the same qualitative behaviours are exhibited. This is illustrated in Figure 3, which depicts equilibrium investment by each party and the resulting perceived fidelity of the cue for a total conflict scenario with emitter payoffs $\Delta T_{1}=-8, \Delta T_{2}=-8$ and $Z=0.75$. Results with $Z=0.6$ and $Z=0.9$ are respectively shown in Figures S6 and S7. The perceived fidelity of the cue at equilibrium when reciprocal evolution occurs is not significantly changed relative to conflict in exclusively one condition; this is in spite of the potential for greater losses to each party for a given value of $Q$ when total conflict pertains.

There is an asymmetry between the value of each condition to both parties; the nondefault condition is infrequent (occurring 20\% of the time) and, thus, parties experience the greatest loss/potential for gain in the more-frequent default condition, which then has the greatest influence in shaping the observed-evolutionary responses. For the parameterisation shown in Figure 3, doubling the value of the non-default condition to the emitter produces only a small increase in equilibrium investment (not shown). In contrast, doubling the value of the default condition produces pronounced increases in 
emitter investment and promotes attenuation for lower values of $\alpha$. This is illustrated in Figure 4 for the emitter payoffs $\Delta T_{1}=-16, \Delta T_{2}=-8$ and $Z=0.75$. Figures S8 and S9 illustrate results for this payoff scenario when $Z=0.6$ and $Z=0.9$ respectively. In spite of the increase in emitter investment, the equilibrium value of $Q$ during reciprocal evolution remains unchanged relative to Figure 3. As noted previously, the equilibrium value of $Q$ when both parties are selected is determined by the incentives and constraints acting upon the perceiver; it is unlikely that the emitter can substantively influence this value so long as it is also beneficial for the perceiver to invest. However, while there is no perceptible decrease in the equilibrium value of $Q$ between Figures 3 and 4, the increased emitter investment in Figure 4 does translate into an extremelysmall decrease in the value of $Q$ (from between three to five decimal places, depending on the parameter combination) and the greatest differences are observed where $\beta=1$ and anti-attenuation does not evolve, whilst the smallest differences occur when antiattenuation is also favoured. The mismatch between the extent of the increase in emitter investment and the change in the final value of $Q$ draws attention to (i) the difficulty in further reducing the value of $Q$ as it approaches the emitter's preferred value and (ii) the difficulty in attaining any further decrease in the value of $Q$ in the presence of anti-attenuation. The selection pressure acting on the emitter must be considerable (as in Figure 4) if the increasingly- marginal returns on further attenuation are to be beneficial.

If the value of the default condition is instead doubled for the perceiver as opposed to the emitter, then there is a pronounced increase in perceiver investment for all values of $Z$ and $\beta$ and the perceiver will evolve anti-attenuation for lower values of $\alpha$ than 
previously. This is illustrated for a total conflict scenario in Figure 5, with the emitter payoffs $\Delta T_{1}=-8, \Delta T_{2}=-8$, the perceiver payoffs $\Delta W_{1}=16, \Delta W_{2}=8$ and $Z=0.75$. Figures S10 and S11 respectively illustrate outcomes for $Z=0.6$ and $Z=0.9$. Elevated perceiver investment translates into a reduction in emitter investment relative to Figure 3, which utilised the same emitter payoffs, and equilibrium values of $Q$ which are closer to $Z$. Thus, as indicated earlier, the greater the potential losses to the perceiver resulting from a decrease in the availability of useful information, the less tolerant of any reduction in the perceived fidelity of this information the perceiver becomes. However, the perceiver is not able to completely obviate the value of what suppression the emitter is able to achieve; even for environments amenable to the perception of the cue $(\beta=5)$, the emitter evolves to a non-zero equilibrium.

\section{Discussion}

There are a huge number of social situations where one party's interests are damaged by another party gaining information about the focal individual. These two parties might be potential mates, competitors, parents, parent and offspring, or predator and prey (to name a few). In such situations, the focal individual might be selected to mask that information, and the other party selected to resist this masking. In this paper, we explore the evolution of such a situation in a very general setting. Our simulations have allowed us to draw a number of conclusions.

To summarise, emitters are selected to attenuate cues when the costs arising from the perception of the cue accrue sufficiently often and are of adequate magnitude. The rate at which costs accrue depends upon the frequency of the conflict and the intrinsic 
informational value of the cue in the absence of attenuation. The less frequent the conflict, or the less reliable the cue, the weaker the selection in favour of attenuation becomes. Hence, a conflict with a small cost will select for attenuation if it arises frequently and the cue reliably elicits the incorrect (from the perspective of the emitter) response from the perceiver, while a rare conflict must carry a higher cost before attenuation is favoured; there is little value in suppressing a cue that is not consistently damaging. The extent to which a given cue can be attenuated depends upon the interaction between (i) the ease of attenuation, (ii) the payoffs available to the emitter, (iii) the intrinsic informational value of the cue and (iv) the response of the perceiver. The first three factors create a ceiling on emitter investment beyond which further investment ceases to be beneficial and so impose an absolute lower limit on the perceived fidelity of the cue. Thus, even in a scenario for which the perceiver is never selected to invest, the emitter is unlikely to be able to fully suppress the cue unless attenuation is decidedly cost-effective. Investment in anti-attenuation by the perceiver lowers the ceiling on emitter investment and maintains the perceived fidelity of the cue at a greater value. The perceiver is selected to invest where attenuation meaningfully encroaches upon the perceiver's rewards; the greater the stakes, the less tolerant of attenuation the emitter becomes. For all three considered conflicts, there were three classes of outcome. First, no evolution occurs. This can be considered a victory to the perceiver; the prevailing conditions do not facilitate the evolution of attenuation and the perceiver will always be able to extract the full informational value of the cue. Second, attenuation evolves but the perceiver is not selected to respond. This represents a victory to the emitter, but is generally limited to scenarios where the extent of attenuation is too low to trigger a response from the perceiver; these cases tend to be constrained to conditions where the conflict has limited value to the perceiver, or 
additional investment in detecting and recognising the cue is inefficient. Finally, reciprocal evolution occurs and both attenuation and anti-attenuation evolve. Neither party achieves its favoured outcome in this instance but, depending upon the incentives acting upon the perceiver, it is possible for one party to have an overriding influence upon the final perceived fidelity of the cue: the greater the selection upon the perceiver to maintain the perceived fidelity of the cue, the more the final outcome resembles the perceiver's optimum.

Our modelling approach assumes that the anti-attenuator trait is able to somehow reconcile the information content of the attenuated signal by partially 'recovering' the intrinsic information content of the cue. In the flycatcher example, this was done by investing a certain amount of time to uncover the eggs. However, this is not the only way in which an attenuator can be countered. For example, the anti-attenuator could directly affect the cost (e.g., alpha) of producing the attenuator itself. While not directly applicable to the flycatcher scenario, one way in which this might work is when the perceiver stays close to the emitter so that it may take the emitter more time to hide the cue. In this case, eq. [2] would not necessarily reflect the mechanism involved and the co-evolutionary dynamic might thus be different. This situation might perhaps imply that when a perceiver is able to invest in a cheap anti-attenuator trait, the emitter simply cannot evolve an attenuator due to prohibitive cost, but more formal elaboration of this different form of anti-attenuation would be a fruitful development of the work described here. It is also possible to imagine different shapes to the functional forms for the link between costs and effectivenesses of attenuators and anti-attenuators to the ones considered here. The most obvious alternative would be one that implied that there had to be an initial substantial investment before there was any payback in 
effectiveness. When this is true for attenuators then we would expect that the parameter space involving no investment in attentuators would grow; where it is true for anti-attentuators then we would expect that this may increase the range of conditions where perceivers tolerate moderate amounts of attenuation without investing in counter-measures. It is important to note that we find the evolution of investment in costly information-masking even in situations where there is partial commonality of interest between the two parties (i.e in the situation explored in the results where there is conflict of interest in one condition but not in the other). That is, we show that costly information-masking can evolve even if there are some conditions (but not all conditions) where emitter and perceiver agree on the preferred action of the perceiver. . This is important, because we would not expect information-masking to be used selectively only in advantageous situations, because then deployment of the mask itself becomes informative. That is, a potential mate could not evolve to only mask his condition when in poor condition if the implementation of the masking can be detected and itself offers a reliable cue of poor quality. Bogaardt \& Johnstone (2016) demonstrated that amplifiers can easily evolve to become informative and become a signal in their own right. Selectively-used attenuators have analogous evolutionary potential, and so we would expect that (unless deployment of attenuators can be undetectable to perceivers) they will be used in the unselective way described here. Importantly, we demonstrate that such attenuators can be evolutionary stable even if they are costly, even if they are sometimes disadvantageous, and even if a perceiver can mount counter-measures to them. As such, we feel that attenuators of cues may be deserving of much more attention from researchers of sensory interactions between animals. 


\section{Acknowledgement}

We thank two reviewers for very helpful comments.

\section{References}

Armbruster, W.S. 1997. Exaptations link evolution of plant-herbivore and plantpollinator interactions: a phylogenetic inquiry. Ecology 78: 1661-1672.

Armbruster, W.S., Antonsen, L. \& Pélabon, C. 2005. Phenotypic selection on Dalechampia blossoms: honest signaling affects pollination success. Ecology 86: 3323-3333.

Bogaardt, L. and Johnstone, R.A., 2016, June. Amplifiers and the origin of animal signals. In Proc. R. Soc. B (Vol. 283, No. 1832, p. 20160324). The Royal Society.

Boulton, R.L. \& Cassey, P., 2012. How avian incubation behaviour influences egg surface temperatures: relationships with egg position, development and clutch size. Journal of Avian Biology, 43: 289-296.

Bradbury, J. W. \& Vehrencamp, S. L. 2000. Economic models of animal communication. Anim. Behav. 59: 259-268. (doi:http://dx.doi.org/10.1006/anbe.1999.1330)

Bradbury, J. W. \& Vehrencamp, S. L. 2011/ Principles of Animal Communication (2nd ed) Sinauer, New York.

Carazo, P., \& Font, E. 2010. Putting information back into biological communication. J. Evol. Biol. 23: 661-669.

Darst C.R., Cummings, M.E. \& Cannatella, D.C. 2006 A mechanism for diversity in warning signals: conspicuousness versus toxicity in poison frogs. PNAS103: 5852-5857

Diekmann, 0., 2002. A beginners guide to adaptive dynamics. Summer School on Mathematical Biology, pp.63-100.

DuRant, S.E., Hopkins, W.A., Hepp, G.R. \& Walters, J.R., 2013. Ecological, evolutionary, and conservation implications of incubation temperature-dependent phenotypes in birds. Biological Reviews, 88(2), pp.499-509. 
Galván, I., \& Sanz, J.J. 2008. The cheek plumage patch is an amplifier of dominance in great tits. Biol. Lett. 4: 12-15.

Guilford, T. \& Dawkins, M.S. 1991. Receiver psychology and the evolution of animal signals. Anim. Behav. 42: 1-14.

Hackett, S., Schaefer, H. M., \& Ruxton, G. D. 2014. Linking signal fidelity and the efficiency costs of communication. J. Evol. Biol. 27: 1797-1810.

Hackett, S., Ruxton, G.D., \& Schaefer, H.M. 2016. Investment in attending to cues and the evolution of amplifiers. J.Evol.Biol. 29: 1131-41.

Hasson, 0. 1989. Amplifiers and the handicap principle in sexual selection - a different emphasis. Proc. R. Soc. Lond. B 235: 383-406.

Loukola, O.J., Laaksonen, T., Seppänen, J.T. and Forsman, J.T., 2014. Active hiding of social information from information-parasites. BMC evolutionary biology, 14(1), p.32.

Maynard Smith J. \& Harper, D. 2003. Animal signals. Oxford University Press, Oxford.

Ord, T.J. \& Stamps, J.A. 2008. Alert signals enhance animal communication in "noisy" environments. PNAS 105: 18830-18835.

Schaefer, H.M. \& Schmidt, V. 2004. Detectability and content as opposing signal characteristics in fruits. Proc. R. Soc. Lond. B 271: 370-373.

Schaefer, H.M. \& Ruxton, G.D. 2012. By-product information can stabilise the reliability of communication. J. Evol. Biol. 25: 2412-2421.

Scott-Phillips, T.C., Blythe, R.A., Gardner, A. \& West, S.A. 2012. How do communication systems emerge? Proc. R. Soc. Lond. B 279: 1943-1949.

Searcy, W.A. \& Nowicki S. 2005. The evolution of animal communication. Princeton University Press, Princeton.

Steiger, S., Schmitt, T. \& Schaefer, H.M. 2011. The origin and dynamic evolution of chemical information transfer. Proc. R. Soc. Lond. B 278: 970-979.

Taylor, P.W., Hasson O. \& Clark, D.L. 2000. Body postures and patterns as amplifiers of physical condition. Proc. R. Soc. Lond. B 267: 917-922. 
Tinbergen, N. 1952. 'Derived' activities; their causation, biological significance, origin, and emancipation during evolution. Q. Rev. Biol. 27: 1-32.

Zahavi, A. 1975. Mate-selection -a selection for a handicap. J. Theor. Biol. 53: 204-214.

Zuk, M., Thornhill, R., Ligon, J.D. \& Johnson, K., 1990a. Parasites and mate choice in red jungle fowl. Am. Zoo. 30: 235-244.

Zuk, M., Johnson, K., Thornhill, R. \& Ligon, J.D., 1990b. Mechanisms of female choice in red jungle fowl. Evolution 44: .477-485.

Zuk, M., Johnson, K., Thornhill, R. \& Ligon, J.D., 1990c. Parasites and male ornaments in free-ranging and captive red jungle fowl. Behav. 114: 232-248.

Zuk, M., Thornhill, R., Ligon, J.D., Johnson, K., Austad, S., Ligon, S.H., Thornhill, N.W. \& Costin, C., 1990d. The role of male ornaments and courtship behavior in female mate choice of red jungle fowl. The Am. Nat. 136: .459-473.

\section{Figure Legends}

Figure 1: Equilibrium investment $\left(K_{i}\right)$ in attenuating and anti-attenuating traits by each party (a) and the perceived fidelity of the cue ( $Q$ ) at equilibrium ( $b$ ) for the emitter payoff scenario $\Delta T_{1}=-8, \Delta T_{2}=8$. The parties disagree with respect to the best perceiver action in the default condition which occurs with probability $P_{1}=0.8$. The perceiver payoffs are $\Delta W_{1}=8, \Delta W_{2}=8$. Equilibrium values are plotted for each value of $\beta$ (the ease with which the focal cue is detected in a given environment) plotted against $\alpha$ (the ease with which the informational value of the cue can be attenuated in a given environment) for $Z=0.75$ where $Z$ represents the intrinsic informational value of the cue. Panel a display the change in the equilibrium cost of attenuation and anti-attenuation (values of $K_{e}$ and $\left.K_{p}\right)$ for each party for a given value of $\beta$ as $\alpha$ increases. In all instances, blue lines denote changes in the relevant emitter variable while red lines indicate changes in the corresponding perceiver variable. Panel b illustrate the corresponding changes in the perceived fidelity of the cue at equilibrium produced by the investment of each party in panel a for a given value of $\beta$ as $\alpha$ increases. 
Figure 2: As for Figure 1 but with conflict in the non-default condition and emitter payoffs $\Delta T_{1}=8, \Delta T_{2}=-40$.

Figure 3: As for Figure 1 but with conflict in both conditions.

Figure 4: As for Figure 3 but with the emitter payoffs $\Delta T_{1}=-16, \Delta T_{2}=-8$.

Figure 5: As for Figure 3 but with the perceiver payoffs $\Delta W_{1}=16, \Delta W_{2}=8$.

This article is protected by copyright. All rights reserved. 


\section{Figures}

Figure 1

\section{Default Conflict $(Z=0.75)$}

Emitter

$\square$ Perceiver
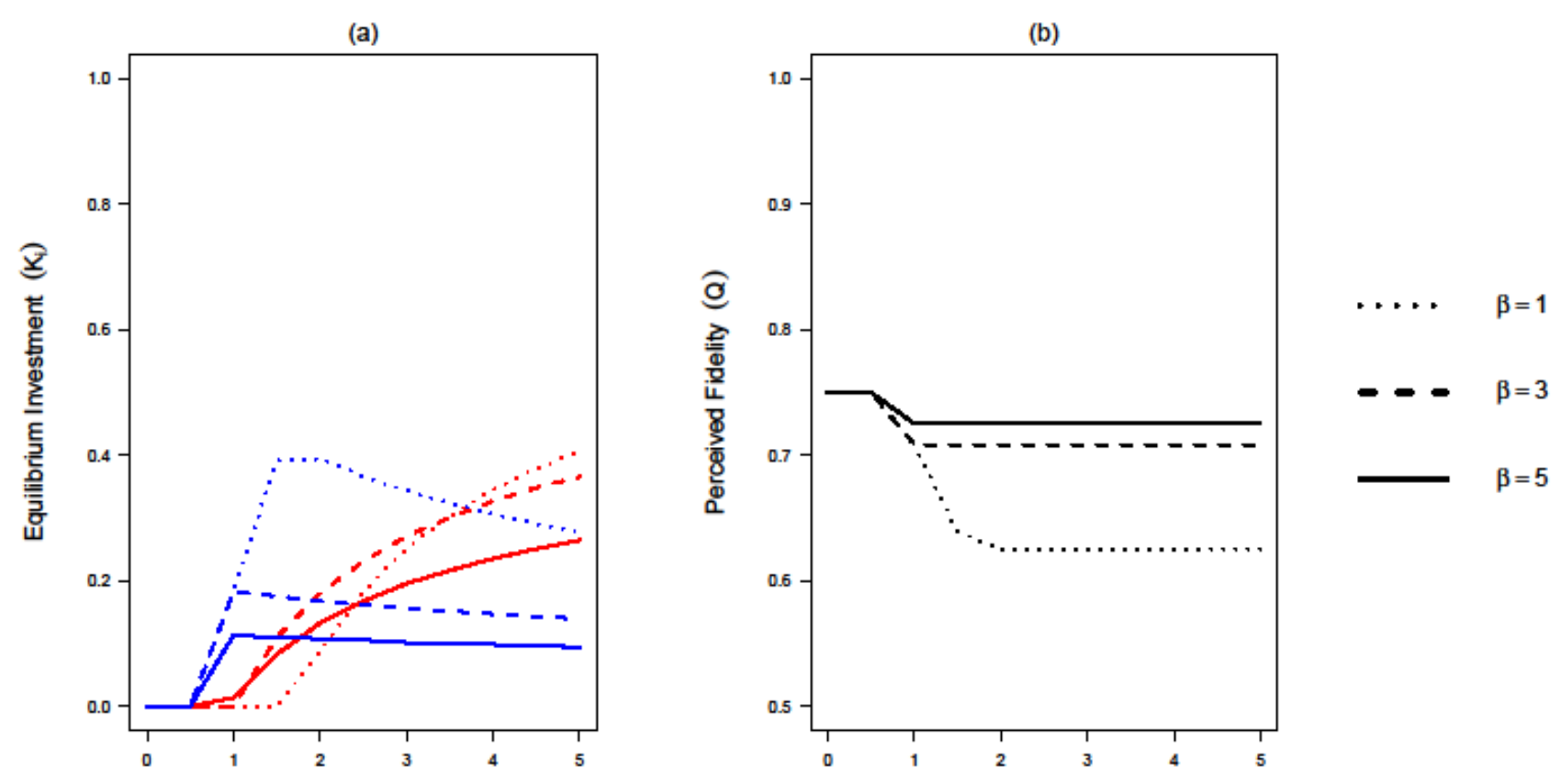

Ease of Attenuation $(\alpha)$

This article is protected by copyright. All rights reserved. 
Figure 2

Non Default Conflict $(Z=0.75)$

Emitter

$\square$ Perceiver

(a)

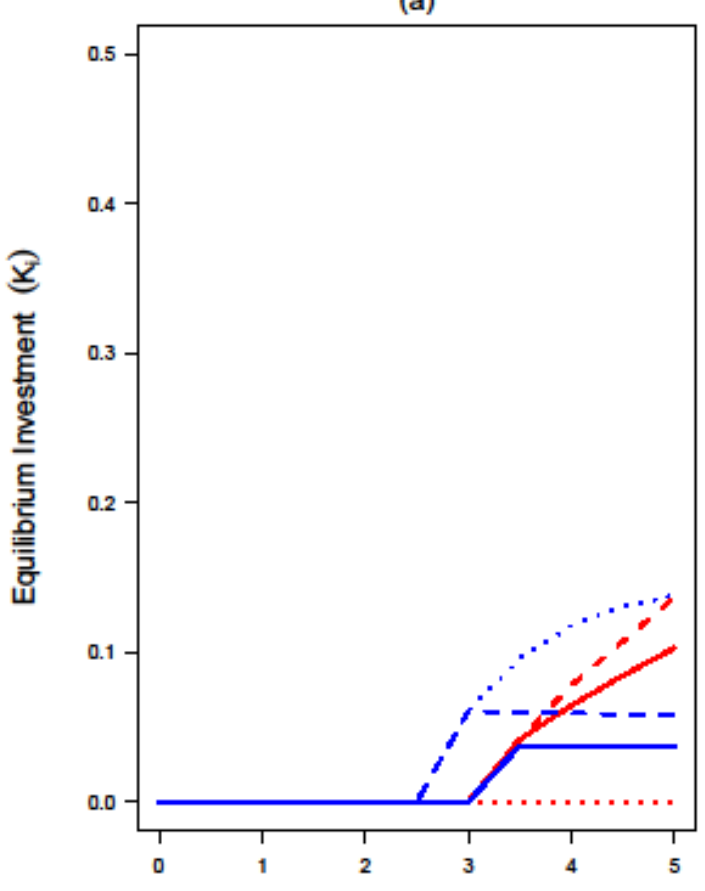

(b)

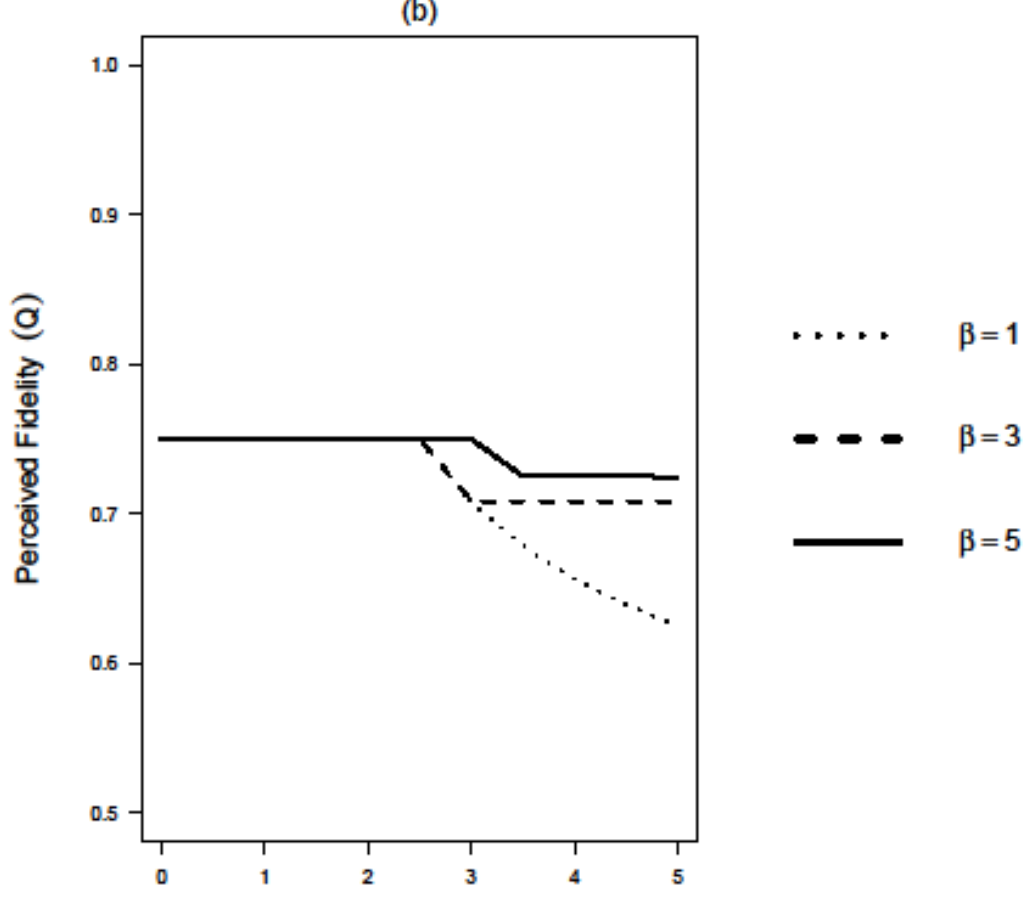

Ease of Attenuation ( $\alpha$ )

This article is protected by copyright. All rights reserved. 
Figure 3

Total Conflict $(Z=0.75)$

Emitter

$\square$ Perceiver

(a)

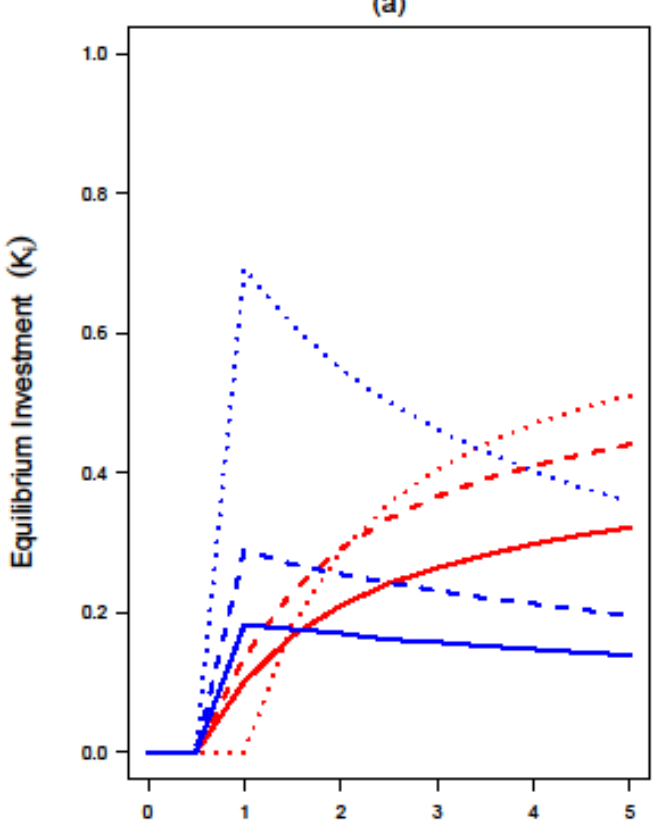

(b)

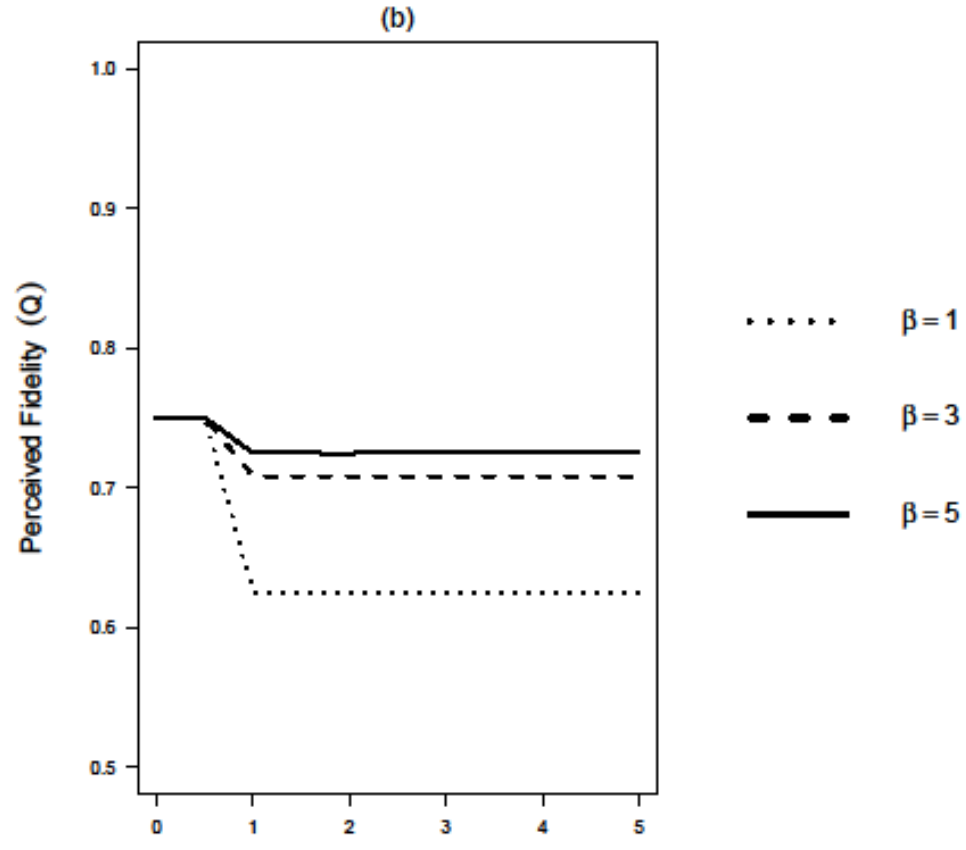

Ease of Attenuation ( $\alpha)$

This article is protected by copyright. All rights reserved. 
Figure 4

Total Conflict ( $Z=0.75$ )

$\square$ Perceiver

(a)

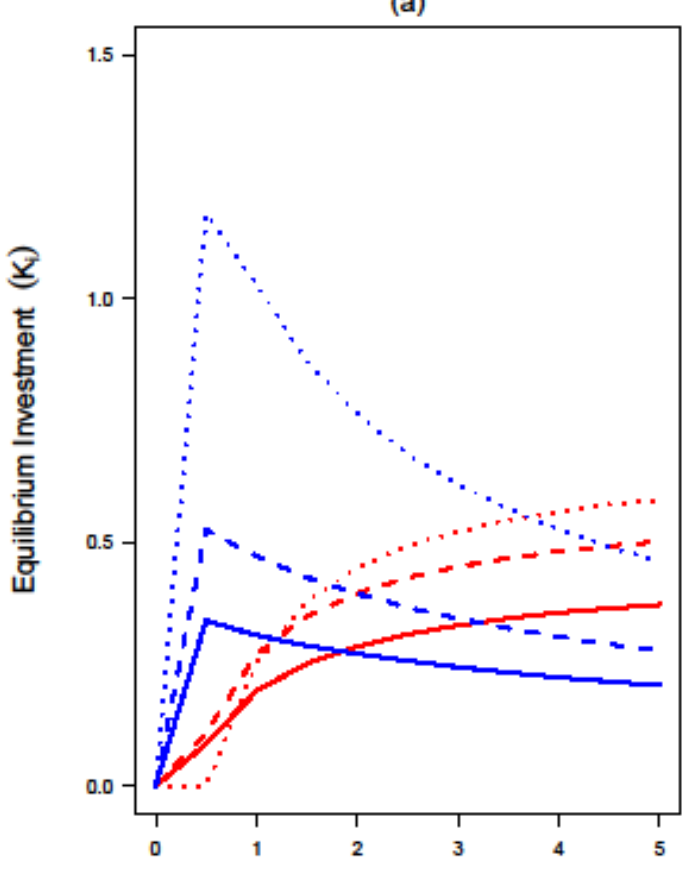

(b)

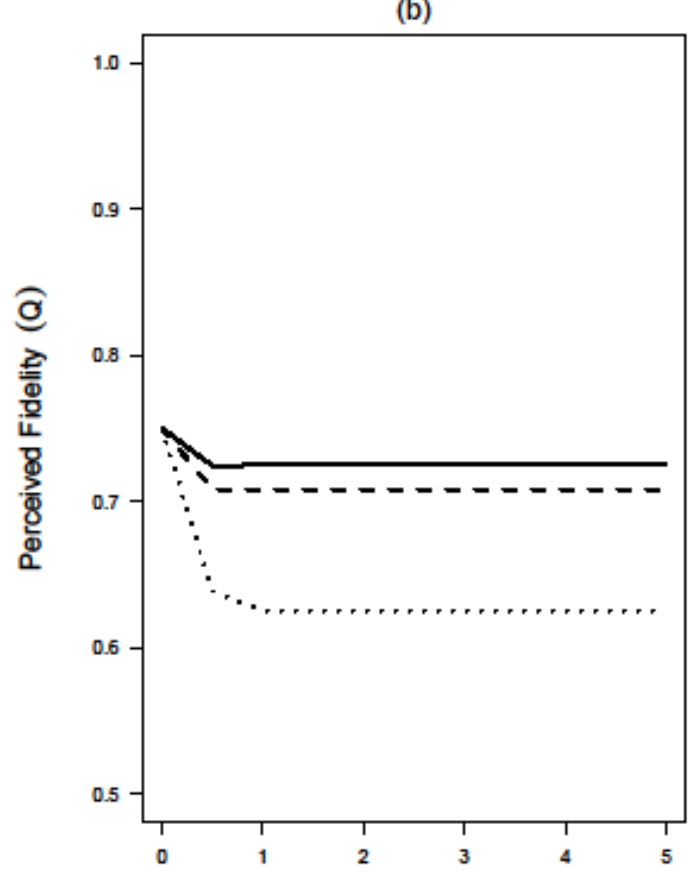

$\cdots \cdots \quad \beta=1$

$-0 \beta=3$

\section{Ease of Attenuation $(\alpha)$}

This article is protected by copyright. All rights reserved. 
Figure 5

Total Conflict $(\mathrm{Z}=0.75)$

Emitter

Perceiver

(a)

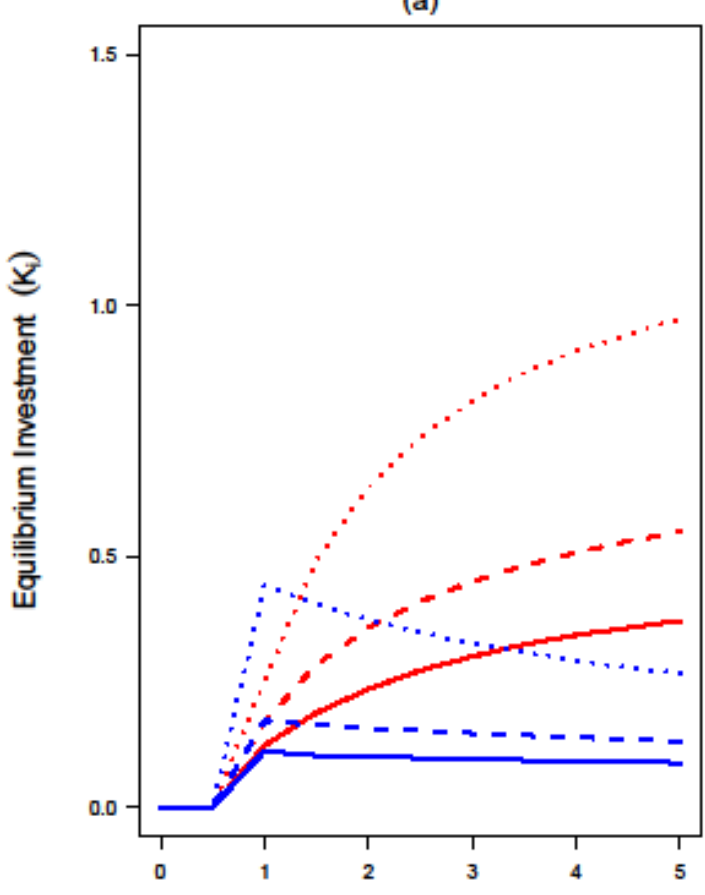

(b)

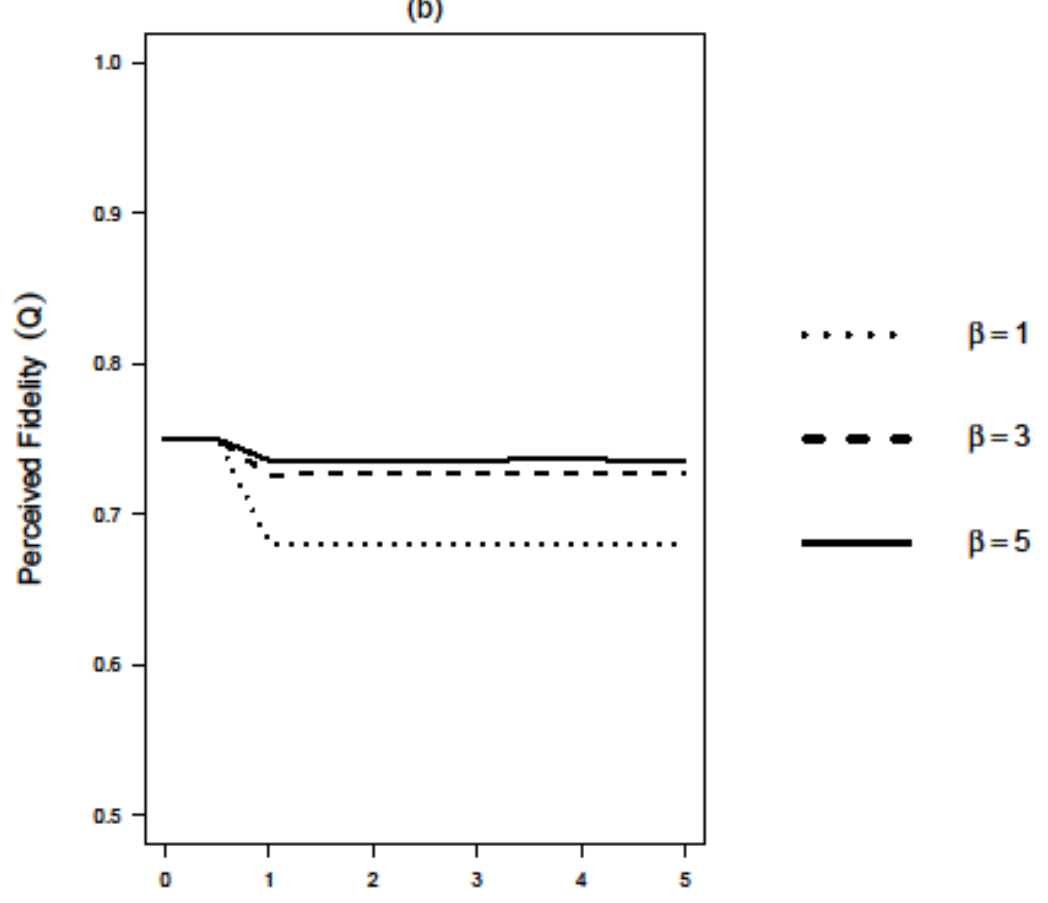

Ease of Attenuation ( $\alpha$ )

This article is protected by copyright. All rights reserved. 